\title{
An Effective Technique of Image Degradation using DWT based Padding Kernel Detection
}

\author{
Varsha Sharma \\ Department of Computer Science \& Engineering \\ Samrat Ashok Technological Institute \\ Vidisha, India
}

\author{
Ajay Goyal \\ Assistant Professor \\ Department of Computer Science \& Engineering \\ Samrat Ashok Technological Institute \\ Vidisha, India
}

\begin{abstract}
Image Filtering is a technique of removing unwanted Noise from image so that the image can be improved in terms of brightness and noise and contrast. Although there are various technique implemented for the Image Degradation and removing Noise level from image such as using Gaussian Blur. The Existing Gaussian Blur technique is an efficient technique which provides more Peak Signal to Noise Ratio and Less Error rate as compare to Zhang Distance and Local Phase Quantization Algorithms. But the technique implemented is not feasible in terms of all images and PSNR and Error Rate, Hence a new and efficient technique is proposed in the paper which is based on the concept of kernel and padding. This work also applies the Haar wavelet Transform for filtering the image in order to reconstruct image which have the noise and blur. The results of this paper show that the proposed method gives the better result from the previous methods. It seems to be that the PSNR, Mean Square Rate and execution time is better in the proposed scheme.
\end{abstract}

\section{Keywords}

DWT Transformation, Haar Wavelet, Debluring, Filtering, Gaussian Blur, Image Degradation.

\section{INTRODUCTION}

Blurring is a form of reduction of the image intensity due to defective image formation process. It can be generally occurred by relative motion between camera and original image. Image restoration is the process of recovering the original image from the infected image. The fundamental task of Image demisting is to de-convolute the infected image with the PSF that exactly describe the distortion. The restoration of blurred image is nothing but improving the quality of blurred or bad images. It also refers for avoiding noise content from the image. It may be occurred by relevant motion between object (scene or image that to be captured) and camera (a device used to capture). Generally, an image can be corrupted using low-pass filters and its noise content. This low-pass filter is used to blur/smooth the image by using convinced operation [1]. Image restoration has been extensively functional for astronomical imaging, medical imaging, remote sensing, microscopy imaging, photography deblurring, among others [2].

Image deblurring is an opposite difficulty which is desire to improve an image that endured from linear degradation. The blurring degradation can be defined as space invariant. This method can be classified into two classes: non blind, in which we know the blurring operator. And blind, in this the blurring operator is unidentified. Image restoration is used to recover an image from deformations to its actual image. It is an intentional process to removes the effects of sensing surroundings. It is the way of improving the original scene image from a corrupted or experiential image using information about its nature. There are two wide categories of image restoration theory such as Image Deconvolution and Blind Image Deconvolution. Image Deconvolution is a linear image restoration difficulty in which the parameters of the actual image are predictable using the experiential or tainted image and a known Point Spread Function (PSF). Blind Image Deconvolution is a more difficult where image recovery is performed with slight or no earlier information of the humiliating PSF. The benefits of Deconvolution are superior resolution and enhanced quality [1].

\subsection{Discrete Wavelet Transform}

A discrete wavelet transform (DWT) is any wavelet transform for which the wavelets are discretely sampled [4]. As with other wavelet transforms, a key advantage it has over Fourier transforms is temporal resolution: it captures both frequency and location information (location in time).Wavelet analysis represents the next logical step: a windowing technique with variable-sized regions. Wavelet analysis allows the use of long time intervals where we want more precise low-Frequency information, and shorter regions [3] where we want high-frequency information.

The Discrete Wavelet Transform (DWT) refers to wavelet transforms for which the wavelets are discretely sampled. A transform which localizes a function both in space and scaling and has some desirable properties compared to the Fourier transform [5]. The transform is based on a wavelet matrix, which can be computed more quickly than the analogous Fourier matrix. Most notably, the discrete wavelet transform is used for signal coding, where the properties of the transform are exploited to represent a discrete signal in a more redundant form, often as a preconditioning [6] for data compression.

\subsection{Image Restoration}

Image Restoration refers to a class of methods that aim to remove or reduce the degradations that have occurred while the digital image was being obtained. Image restoration problems can be quantified precisely [7].

\subsubsection{One Dimensional Discrete Degradation Model}

Suppose we have a one-dimensional discrete signal f(i) of size $A$ samples $\mathrm{f}(0), \mathrm{f}(1), \ldots ., \mathrm{f}(\mathrm{A}-1)$ which is due to a degradation process.

The degradation can be modeled by a one-dimensional discrete impulse response h(i) of size $B$ samples. If we assume that the degradation is a causal function we have the samples

h (0), h (1),...h (B-1). 
We form the extended versions of $\mathrm{f}(\mathrm{i})$ and $\mathrm{h}(\mathrm{i})$, both of size $M \geq A+B-1$ and periodic with period $M$. These can be denoted as $f_{e}(i)$ and $h_{e}(i)$.

For a time invariant degradation process we obtain the discrete convolution formulation as follows

$$
y_{e}(i)=\sum_{m=0}^{M-1} f_{e}(m) h_{e}(i-m)+n_{e}(i)
$$

\subsubsection{Two Dimensional Discrete Degradation Model}

Suppose we have a two-dimensional discrete signal $f(i, j)$ of size $A \times B$ samples which is due to a degradation process [18].

The degradation can now be modeled by a two dimensional discrete impulse response $h(i, j)$ of size $C \times D$ samples.

We form the extended versions of $f(i, j)$ and $h(i, j)$, both of size $M \times N$, where $M \geq A+C-1$ and $N \geq B+D-1$, and periodic with period $M \times N$. These can be denoted as $f_{e}(i, j)$ and $h_{e}(i, j)$.

For a space invariant degradation process we obtain

$$
y_{e}(i, j)=\sum_{m=0}^{M-1} \sum_{n=0}^{N-1} f_{e}(m, n) h_{e}(i-m, j-n)+n_{e}(i, j)
$$

Using matrix notation we can write the following form

$$
\mathbf{y}=\mathbf{H f}+\mathbf{n}
$$

Where $\mathbf{f}$ and $\mathbf{y}$ are $M N$ - dimensional column vectors that represent the lexicographic ordering of images $f_{e}(i, j)$ and $h_{e}(i, j)$ respectively.

\section{LITERATURE SURVEY}

In [8] the edges of the blurred image, the ringing effect can be detected using Canny Edge Detection method and then it can be removed before restoration process. Blind Deconvolution algorithm is applied to the blurred image. It is possible to renovate the original image without having specific knowledge of degradation filter, additive noise and PSF. To get the effective results, the Penalized Maximum Likelihood (PML) Estimation Technique is used with our proposed Blind Deconvolution Algorithm.

They proposed [9] a robust image restoration method using two-dimensional block Kalman filter with colored driving source. This method aims to achieve high quality image restoration for blur and noise disturbance from the canonical state space models with (i) a state equation composed of the original image, and (ii) an observation equation composed of the original image, blur, and noise. The remarkable feature of the proposed method is realization of high performance image restoration without sacrificing original image despite simple image restoration using only Kalman filter algorithm, while many conventional methods based on the Kalman filter theory usually perform the image restoration, using the parameter estimation algorithm of AR (auto regressive) system and the Kalman filter algorithm.
In this [10] work a new two-dimensional method for blind image restoration, based on an $L 1$ regularization cost function is available. A universal gradient algorithm is proposed by using a weak derivative of the absolute value function to handle with the non-differentiable case. Unlike the double regularization (DR) approach, the proposed method uses the $L 1$ estimation and is suitable for blind image restoration under non-Gaussian noise environments.

Common issue in image restoration is ill-posed, so regulation technique [11] is needed to restrict the problem. In this paper the error cost function with adaptive selection of regularization parameter (ASPR) is created in spatial domain, and the conjugate gradiant is evolved to minimize the error cost function. In this frequency domain two conditions are incorporated in the estimation process of the object image and PSF. ASPR method can gain the regularization parameter adaptively according to the border information of the image proposed, which provides assurance the restored image is the most excellent result in the total field Simulation results show that this approach is accurate and reasonable, as well as has a good performance in the uniqueness and convergence of solution.

Non-blind image deconvolution [12] is a process that obtains a sharp latent image from a hazed image when a point spread function (PSF) is known. In other hand, ringing and noise intensification are inevitable artifacts in image deconvolution since perfect PSF estimation is impossible. The traditional regularization to decrease these artifacts cannot preserve image details in the de-convolved image when PSF estimation error is huge, so burly regularization is required.

They apply Bayesian blind source separation (BSS) [13] from noisy convolutive mixtures to jointly separate and restore source images degraded through unknown blur operators, and then linearly mixed. They found that this problem arises in several image processing applications, among which there are some interesting instances of degraded document analysis. In particular, the convolutive mixture model is proposed for describing multiple views of documents affected by the overlapping of two or more text patterns.

A method for blind image deblurring [14] is presented. The method only makes weak assumptions about the blurring filter and is able to undo a wide variety of blurring degradations. To overcome the ill posedness of the blind image deblurring problem, the method includes a learning technique which initially focuses on the main edges of the image and gradually takes details into account. A new image prior, which includes a new edge detector, is used. The method is able to handle unconstrained blurs, but also allows the use of constraints or of prior information on the blurring filter, as well as the use of filters defined in a parametric manner. Furthermore, it works in both single-frame and multi-frame scenarios. The use of constrained blur models appropriate to the problem at hand, and/or of multi-frame scenarios, generally improves the deblurring results.

\section{PROPOSED METHODOLOGY}

The paper focuses on restoration of blurry and noisy images. Blur and noise are the main source of degradations. To minimize or remove the effect of these degradations, proposed method introduces the concept of the two things i.e., kernel and padding. The method is called as Hybrid kernel padding. The proposed methods are explained in the following three processes: 
1. Load the image.

2. Apply the Gaussian blur kernel and find the size of the kernel (determines the amount of blur)

3. Choose the variance of the Gaussian function. A large variance will result in more blur.

4. $\mathbf{F}\left\{\mathbf{f}^{*} \mathbf{g}\right\}=\mathbf{F}\{\mathbf{f}\} . \mathbf{F}\{\mathbf{g}\} f$ is the image, $g$ is the blur kernel, and $f^{*} g$ gives us the blurred image.

5. In order for the DWT of the kernel to be multiplied with the DWT of the image, the kernel must be placed into an image of the same size as the original image. The kernel can be placed anywhere in the image; it doesn't really matter. After the kernel is placed into a new image, 2-D DWTs of the kernel image and original image are taken. The DWT of the kernel is then modified so that there are no zero values. This is not important now because we are performing a multiplication with the DWT of the kernel, but later, when we deblur, we will need to perform a division, and any zero values in the DWT of the kernel will prompt a warning. To ensure that we are performing symmetric operations, we should remove the zero values from the DWT of the kernel first. After the zeros are removed from the DWT of the kernel, a multiplication is performed with the DWT of the original image. This is a point wises multiplication, not a matrix multiplication, which would give much different results and probably an error, in this case. Next, the DWT of this product is transformed back into the image domain with an inverse 2-D DWT. This procedure follows the second equation mentioned earlier.

6. Before applying DWT apply padding to blur the image.

7. Apply reverse methodology to unblur image.

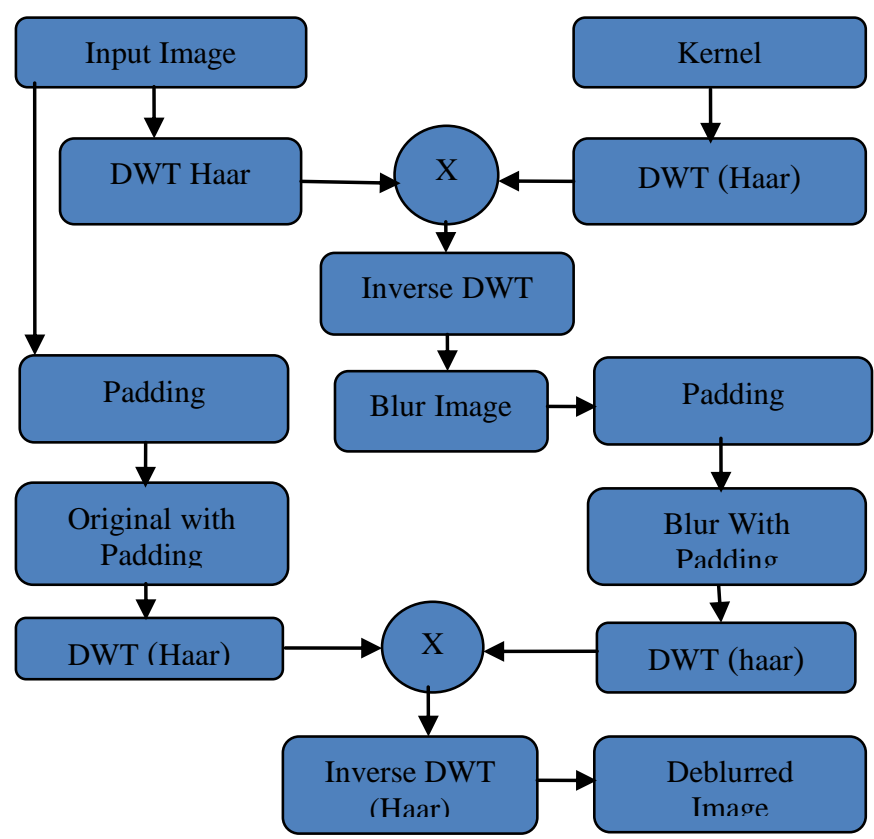

Fig .1 Outline of Proposed Work

\subsection{Components of Proposed Architecture}

\subsubsection{Kernel:}

The kernel tells the location and amount of blurring present in the image. Blurring is just spreading out the information from each point into the surrounding points. The function that accomplished this task in the image domain is called 'convolution'. The kernel is calculate by the following formulae by setting the values of variance(s) and mean (m)

Kernel image $=\left(1 /\left(2 * \mathrm{pi}^{*} \mathrm{~s}^{\wedge} 2\right)\right) * \exp \left(-\left((\mathrm{X}-\mathrm{m}) \mathbf{.}^{\wedge} \mathbf{2}+(\mathrm{Y}-\right.\right.$ $\left.\left.\mathrm{m}) \mathbf{\wedge}^{\wedge} 2\right) /\left(2 * \mathrm{~s}^{\wedge} \mathbf{2}\right)\right)$

\subsubsection{Dwt Haar Transform}

Now DWT Haar is applied on input image and kernel image.DWT decomposes the input image into frequency subbands by using multiple Haar wavelet filters and pyramid decomposition method. The Haar wavelet filter is apply on image and kernel level by level. The method use level 2, so first DWT Haar is apply on kernel image and input image at one level and the image is further decomposed into four subbands : LL, LH,HL,HH referred as Low-Low, Low-High, High-Low and High-High. The Low-low subband gives an approximation of the image which is to be used to apply on. And the remaining three sub bands i.e. LH, HL, HH gives the missing details of the image. On the second level, the LL subbands are further decomposed into four subbands that is LL, LH, HL and HH as shown in fig. At each level, image is decomposed into low and high frequency subbands and hence the image must be a here $\mathrm{n}$ is the no. of level used.

After applying the Haar wavelet on kernel and input image, multiply both the images. Point wise multiplication is performed, not matrix multiplication. To obtain the result in image domain apply Inverse Haar Wavelet Transform and the blurred image is obtained.

\subsubsection{Padding}

There could be some aliasing effect in the blurred image edges. To remove the effect of aliasing, image need to be pad. Padding is applied on original image as well as on blur image with the size of the kernel i.e. 31 in this case.

DWT Haar is performed on original image with pad and blur image with pad for filtration purpose and then multiply together. Inverse DWT Haar is applying in order to get the result in image domain i.e. (2-D).

Decide what values you want to use to pad (zeroes, average value, repeated values, etc.) Pad the image by at least the size of your kernel (the non-zero part) Pad further to nearest power of 2 if using the FFT (may be required depending on the implementation) Fourier Transform, multiply, inverse Fourier Transform Crop back to the original size

\section{Algorithm}

1. Take an input image consisting of blur or noise.

2. Detect the kernel region of the original image.

3. Blur the image if required using kernel image and DWT transformation.

4. Apply padding of the original image.

5. Restored the image from the padding image and kernel image.

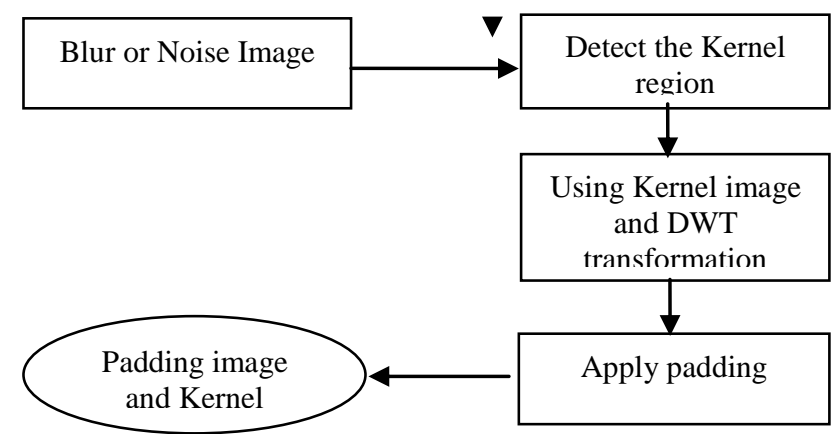

Fig. 2 Flow Chart of Propose work 
Take an input image which may contain corrupt image or blurred image. First we will find the kernel of the original image by taking having kernel size 31 and by taking the level of the kernel size as 3 we will find the kernel image of the original image by calculating

Kernel image $=\left(1 /\left(2 * \mathrm{pi}^{*} \mathrm{~s}^{\wedge} 2\right)\right)^{*} \exp \left(-((\mathrm{X}-\mathrm{m}))^{\wedge} 2+(\mathrm{Y}-\right.$ m).^2)/(2*s^2));

Aftesr finding the kernel of the image we will apply DWT algorithm of level 2 in which we have applied HAAR wavelet transformation and can be given as:

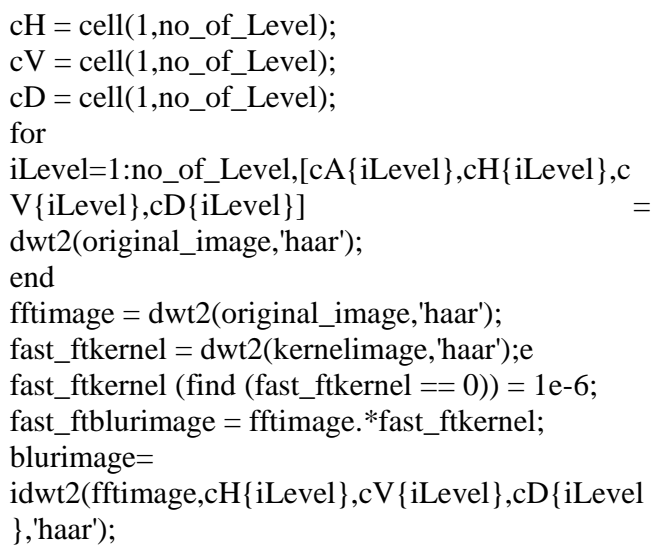

Then we have applied padding of the image so that the image gets leveled where the blurring is more. Then we will apply inverse dwt transformation to get the unblurring of the padding the image.

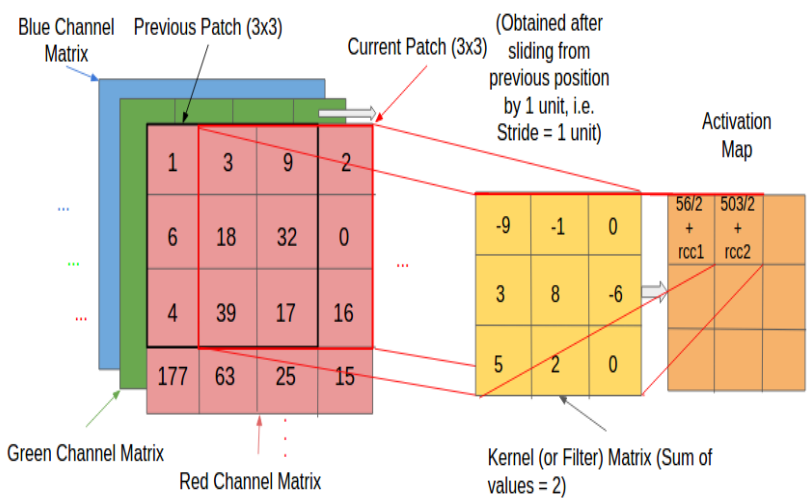

Fig.3 Example of Kernel padding in Image

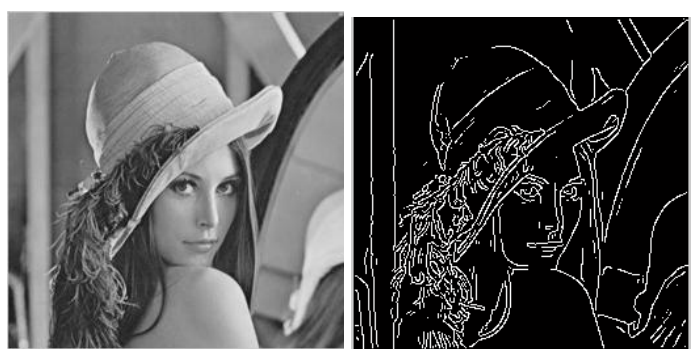

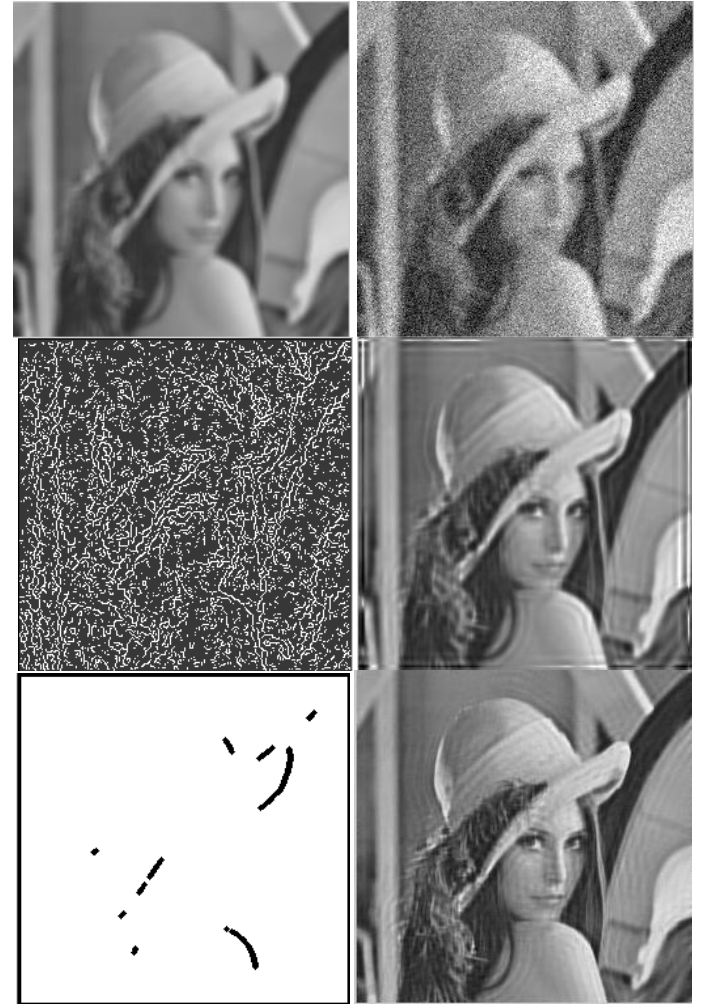

Fig.4 Debluring Image Degradation using Proposed Work

\section{RESULT ANALYSIS}

The Table [1] shown below is the analysis and comparison of Existing Image Degradation and proposed work on the basis of Recognition Rate. The Proposed Methodology provides high Recognition Rate.

Table 1.Analysis of Recognition Rate

\begin{tabular}{|c|c|c|}
\hline & \multicolumn{2}{|c|}{ Recognition Rate } \\
\hline SNR & Gaussian Blur & $\begin{array}{c}\text { Proposed } \\
\text { Work }\end{array}$ \\
\hline 60 & 100 & 100 \\
\hline 40 & 100 & 100 \\
\hline 20 & 74 & 82 \\
\hline 10 & 30 & 43 \\
\hline 5 & 26 & 38 \\
\hline 0 & 21 & 32 \\
\hline
\end{tabular}

The Figure [5] shown below is the analysis and comparison of Existing Image Degradation and proposed work on the basis of Recognition Rate. The Proposed Methodology provides high Recognition Rate. 


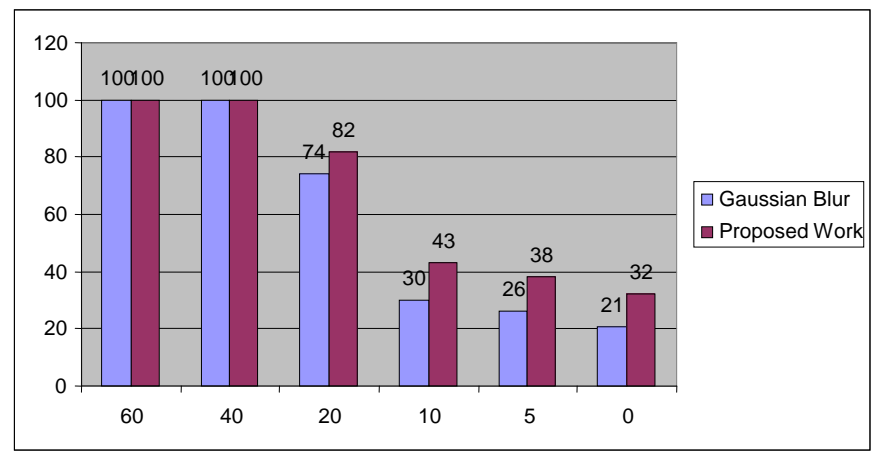

Fig.5 Comparison of Recognition Rate(\%)

The Table [2] shown below is the analysis and comparison of Existing Image Degradation and proposed work on the basis of Mean Error Rate and Standard Deviation. The Analysis done here is for Noisy Free Images.

Table 2.Analysis of various Schemes on Noise Free Templates

\begin{tabular}{|c|c|c|}
\hline Method & Mean Error & Std. \\
\hline CC & 42.53 & 22.22 \\
\hline ZD & 0.16 & 0.08 \\
\hline ID & 0.39 & 0.2 \\
\hline Proposed & 0.12 & 0.05 \\
\hline
\end{tabular}

The Figure [6] shown below is the analysis and comparison of Existing Image Degradation and proposed work on the basis of Mean Error Rate and Standard Deviation. The Analysis done here is for Noisy Free Image.

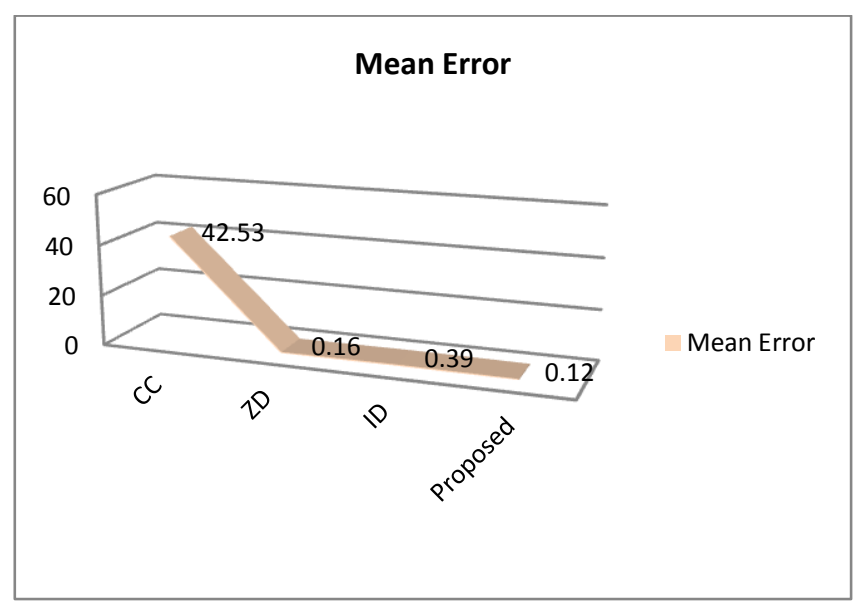

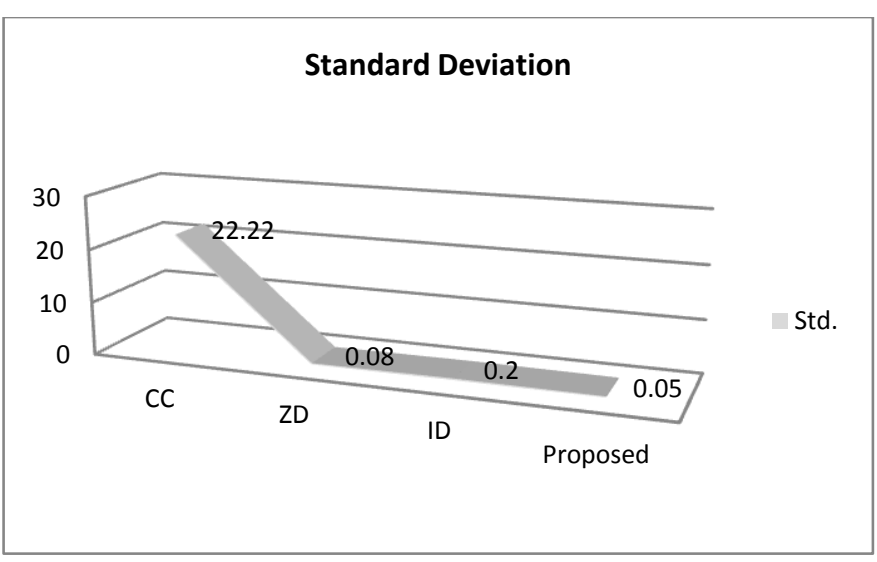

Fig.6 Comparison of Mean Error and Standard Deviation (Std)

The Table[3] shown below is the analysis and comparison of Existing Image Degradation and proposed work on the basis of Mean Error Rate and Standard Deviation.

The Analysis done here is for Noisy Images.

Table 3. Analysis of various Schemes on Noisy Templates

\begin{tabular}{|c|c|c|}
\hline Method & Mean Error & Std. \\
\hline CC & 41.24 & 21.55 \\
\hline ZD & 43.99 & 22.98 \\
\hline ID & 0.9 & 0.47 \\
\hline Proposed & 0.5 & 0.36 \\
\hline
\end{tabular}

The Figure [7] shown below is the analysis and comparison of Existing Image Degradation and proposed work on the basis of Mean Error Rate and Standard Deviation(Std). The Analysis done here is for Noisy Images.

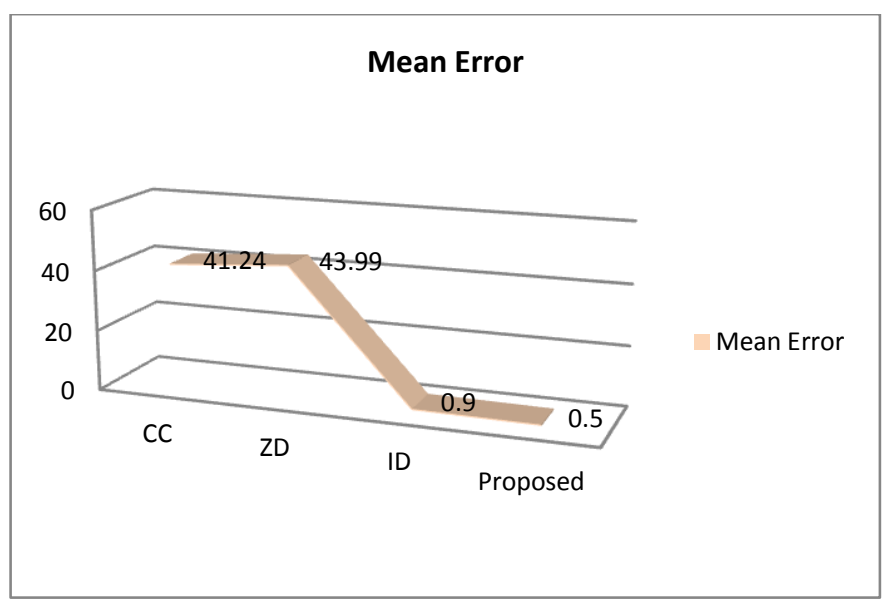




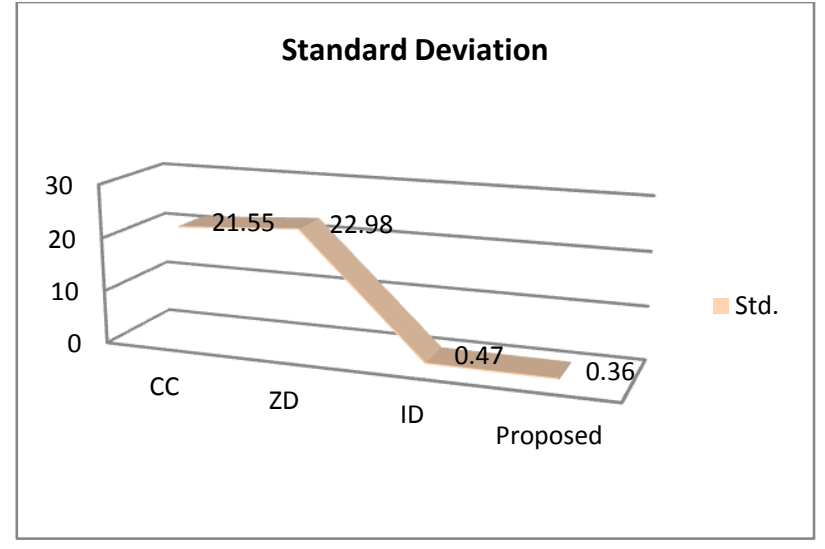

Fig.7 Comparison of Mean Error and Standard Deviation (Std)

\section{CONCLUSION}

Image restoration is the process of removing the blurry or noisy effect from the image. Here in this paper an efficient technique for the restoration of images is proposed. The result parameters shows that the efficiency of the proposed technique. The Error rate of the techniques is less as compared to the previous techniques. Also the blurry and noise effect can be effectively reduced using our proposed work.

In the future some enhanced can be implemented for the deblurryness of the image. The HDR images also contains some noise and blur which is difficult to remove using such techniques that are implemented here. Hence our future work will be on the removal of noisy and blurry HDR images.

\section{REFERENCES}

[1] S.Ramya, T.Mercy Christial "Restoration of blurred images using Blind Deconvolution Algorithm", 2011 International Conference on Emerging Trends in Electrical and Computer Technology (ICETECT), pp. 496 - 499, March 2011

[2] Jinlian Zhuang and Youshen Xia "A Two-Dimensional Iterative Algorithm for Blind Image Restoration based on An L1 Regularization Approach", 2010 3rd International Congress on Image and Signal Processing (CISP2010), pp. $51-55,2010$.

[3] E. Ordentlich, M. Weinberger, G. Seroussi,"A lowcomplexity modeling approach for embedded coding of wavelet coefficients", In Proc. IEEE Data Compression Conf., Snowbird, UT, pp. 408-417, Mar. 1998.
[4] W.A.Pearlman,"Performance bounds for sub-band codes", Chapter 1 in Sub-band Image Coding, J. W. Woods and Ed. Klvwer. Academic Publishers, 1991.

[5] A.Said, W.A. Pearlman,"A new, fast and efficient image codec based on set partitioning in hierarchical trees", IEEE Trans. on Circuits and Systems for Video Technology 6, pp. 243-250, June 1996.

[6] P.Schelkens, "Multi-dimensional wavelet coding algorithms and implementations", Ph.D dissertation, Department of Electronics and Information Processing, Vrije Universitie Brussel, Brussels, 2001.

[7] 'Digital Image Restoration', by M.R. Banham and A.K. Katsaggelos, IEEE Signal Processing Magazine, pp. 27-41, March 1997.

[8] S.Ramya, T.Mercy Christial "Restoration of blurred images using Blind Deconvolution Algorithm", International Conference on Emerging Trends in Electrical and Computer Technology (ICETECT), pp $496-499,2011$.

[9] Ryu Nagayasu, Naoto Hosoda, Nari Tanabe, Hideaki Matsue, Toshihiro Furukawa "Restoration Method For Degraded Images Using Two-Dimensional Block Kalman Filter With Colored Driving Source”, IEEE Digital Signal Processing Workshop and IEEE Signal Processing Education Workshop (DSP/SPE), pp. 151 156, 2011.

[10] Sun qi, Hongzhi Wang, Lu wei, “ An iterative blind deconvolution image restoration algorithm based on adaptive Selection of regularization parameter", Third International Symposium on Intelligent Information Technology Application (IITA 2009), pp. 112 - 115, 2009.

[11] Jong-Ho Lee, Yo-Sung H "High-quality non-blind image deconvolution with adaptive regularization", Journal of Visual Communication and Image Representation, Volume 22, Issue 7, pp. 653-663, 2011

[12] Anna Tonazzini, Ivan Gerace, and Francesca Martinelli "Multichannel Blind Separation and Deconvolution of Images for Document Analysis", IEEE Transactions on Image Processing, Vol. 19, No. 4, pp. 912-925, April 2010.

[13] Mariana S. C. Almeida and Luís B. Almeida "Blind and Semi-Blind Deblurring of Natural Images", IEEE Transactions on Image Processing, Vol. 19, No. 1, pp. 36-52, January 2010. 